ARTICLE

Received 8 Jul 2014 | Accepted 8 Aug 2014 | Published 19 Sep $2014 \quad$ DOl: 10.1038/ncomms5951

\title{
Kinetochores require oligomerization of Dam1 complex to maintain microtubule attachments against tension and promote biorientation
}

Neil T. Umbreit ${ }^{1}$, Matthew P. Miller ${ }^{2}$, Jerry F. Tien ${ }^{1}$, Jérôme Cattin Ortolá1, Long Gui ${ }^{3}$, Kelly K. Lee ${ }^{3}$, Sue Biggins ${ }^{2}$, Charles L. Asbury ${ }^{4} \&$ Trisha N. Davis ${ }^{1}$

Kinetochores assemble on centromeric DNA and present arrays of proteins that attach directly to the dynamic ends of microtubules. Kinetochore proteins coordinate at the microtubule interface through oligomerization, but how oligomerization contributes to kinetochore function has remained unclear. Here, using a combination of biophysical assays and live-cell imaging, we find that oligomerization of the Dam1 complex is required for its ability to form microtubule attachments that are robust against tension in vitro and in vivo. An oligomerization-deficient Dam1 complex that retains wild-type microtubule binding activity is primarily defective in coupling to disassembling microtubule ends under mechanical loads applied by a laser trap in vitro. In cells, the oligomerization-deficient Dam1 complex is unable to support stable bipolar alignment of sister chromatids, indicating failure of kinetochoremicrotubule attachments under tension. We propose that oligomerization is an essential and conserved feature of kinetochore components that is required for accurate chromosome segregation during mitosis.

\footnotetext{
${ }^{1}$ Department of Biochemistry, University of Washington, Seattle, Washington 98195, USA. ${ }^{2}$ Division of Basic Sciences, Fred Hutchinson Cancer Research Center, Seattle, Washington 98109, USA. ${ }^{3}$ Department of Medicinal Chemistry, University of Washington, Seattle, Washington 98195 , USA. ${ }^{4}$ Department of Physiology and Biophysics, University of Washington, Seattle, Washington 98195, USA. Correspondence and requests for materials should be addressed to T.N.D. (email: tdavis@uw.edu).
} 
A ccurate chromosome segregation is mediated by kinetochores, which physically link replicated chromosomes to the bipolar mitotic spindle. During mitosis, kinetochores utilize microtubule tip dynamics to produce mechanical forces required for chromosome biorientation and segregation. In the budding yeast Saccharomyces cerevisiae, the heterodecameric Daml complex is responsible for coupling kinetochores to microtubule ends and for maintaining these attachments during biorientation, when tension is applied across the kinetochores assembled on sister chromatid pairs ${ }^{1-3}$. In vitro, the Dam1 complex spontaneously assembles into oligomeric rings that encircle microtubules, and it has long been hypothesized that the ability to adopt this ring conformation is critical for its roles in kinetochore-microtubule attachment ${ }^{4-7}$. However, ring formation is not required for the Dam 1 complex to track with disassembling microtubule ends in the absence of applied tension in vitro $^{8}$. Thus, it remains unclear whether oligomerization is required for any specific functions of the Dam1 complex, or even if oligomeric rings exist at kinetochores during mitosis.

The Dam 1 complex is required for kinetochores to sustain attachments to microtubule ends against high forces, and to modify microtubule tip dynamics to promote microtubule growth $^{9-11}$. It binds to kinetochores via direct interaction with the Ndc80 complex on microtubules ${ }^{12,13}$. Here to address whether oligomerization is important for any of these functions, we used a Dam1 subcomplex that lacks the ability to assemble into oligomeric rings on microtubules, as determined by negative-stain electron microscopy ${ }^{14}$. Deletion of the gene encoding Hsk3 causes the remaining nine components of the Dam1 complex to split into two subcomplexes. One of these contains six proteins-Dam1, Duo1, Spc34, Spc19, Dad1 and Dad3-and binds microtubules in vitro. This is the 'Oligomerization-Deficient' Dam1 ${ }^{\mathrm{OD}}$ complex used in this study. The other three components (Ask1, Dad2 and Dad4) form the second subcomplex, which does not bind microtubules ${ }^{14}$.

Using in vitro biochemical and biophysical assays, we found that the Dam $1^{\mathrm{OD}}$ complex represents a clean separation of functions: it retains an intact microtubule-binding site, but is deficient in oligomerization. We identified a striking defect of the Dam $1^{\mathrm{OD}}$ complex in coupling to disassembling microtubule tips against applied tension. Finally, we asked if oligomerization of the Dam1 complex is required in vivo. As every component of the Dam 1 complex is essential for viability ${ }^{15}$, an Hsk3-degron system was used to transiently generate the Dam ${ }^{\mathrm{OD}}$ complex in yeast cells. Consistent with our in vitro observations, kinetochoremicrotubule attachments in these cells failed during bipolar alignment of sister chromatids, resulting in mitotic arrest. Our work provides compelling evidence for a specific and essential function of oligomerization at the kinetochore-microtubule interface.

\section{Results}

Microtubule binding and oligomerization are separable. Miranda et al. ${ }^{14}$ previously reported a stable, six-protein Dam1 subcomplex that co-sediments with microtubules, but is unable to assemble into oligomeric rings (Supplementary Fig. 1). We corroborated and expanded these findings to verify the fact that this Oligomerization-Deficient $\mathrm{Dam} 1^{\mathrm{OD}}$ complex retains a functional microtubule-binding domain in the absence of oligomerization. Using total internal reflection fluorescence (TIRF) microscopy ${ }^{8,12}$, we imaged single wild-type (Dam1 $1^{\mathrm{WT}}$ ) and Dam 1 OD complexes tagged with Dad1-green fluorescent protein (GFP) as they bound to microtubules (Fig. 1a). Distributions of GFP fluorescence intensity were similar for the
Dam $1^{\text {WT }}$ and Dam $1^{\text {OD }}$ complexes, and the magnitude of single photobleach steps in our imaging conditions suggests that these distributions derive from single GFP molecules (Fig. 1b). Thus, at the low concentrations (5-40 pM) required for single-molecule imaging, both Dam 1 WT and Dam $1{ }^{\mathrm{OD}}$ complexes exist primarily as monomers. Residence time distributions and standard diffusion plots showed that the Dam1 $1^{\text {WT }}$ and $\mathrm{Dam} 1^{\mathrm{OD}}$ complexes exhibit similar microtubule-binding properties (Fig. 1c,d). We determined single-molecule mean residence times of $5.3 \pm 0.5$ (error of the mean) and $7.0 \pm 0.5 \mathrm{~s}$, and diffusion constants of $0.021 \pm 0.001$ (s.d. of the fit) and $0.017 \pm 0.001 \mu \mathrm{m}^{2} \mathrm{~s}^{-1}$, for Dam1 ${ }^{\mathrm{WT}}$ and $\mathrm{Dam}^{\mathrm{OD}}$ complexes, respectively. Thus, at the single-molecule level, the oligomerization-deficient Dam1 complex binds microtubules in a manner that is similar to the wild-type (WT) complex.

At a higher concentration $(2 \mathrm{nM})$ that supports the formation of oligomeric rings ${ }^{12}$, Dam $1^{\mathrm{WT}}$ complex formed a punctate distribution along microtubules, consistent with its cooperative binding ${ }^{8}$ and its assembly into oligomers (Supplementary Fig. 2a). Despite their similar behaviour at the single-molecule level, $2 \mathrm{nM}$ Dam $1{ }^{\mathrm{OD}}$ complex decorated the microtubule lattice at a much lower density relative to $2 \mathrm{nM}$ Dam $1^{\mathrm{WT}}$ complex (Supplementary Fig. 2b). Instead, $20 \mathrm{nM}$ Dam $1{ }^{\text {OD }}$ complex was needed to achieve a binding density on microtubules comparable to that of $2 \mathrm{nM}$ WT, and the decoration by Dam $1{ }^{\mathrm{OD}}$ complex appeared more uniform (Supplementary Fig. 2a,b). Thus, the Dam ${ }^{\mathrm{OD}}$ complex is impaired in binding cooperativity, as expected for an oligomerization-deficient species. We visualized individual molecules in these conditions by mixing small ('tracer') quantities of GFP-tagged Dam1 complex with excess un-tagged complex (Supplementary Fig. 2c-e). For Dam $1^{\mathrm{WT}}$ complex, 'tracer' GFP-tagged molecules exhibited long residence times on the microtubule lattice, suggesting their incorporation into oligomers of un-tagged Dam $1^{\mathrm{WT}}$ complexes (Supplementary Fig. 2c). We note that the residence time of 'tracer' Dam 1 WT complexes, $56 \pm 8 \mathrm{~s}$ (Supplementary Fig. 2f), is comparable to the average single-step photobleaching time in our imaging conditions $(51 \pm 16 \mathrm{~s})$. Therefore, this measurement likely underestimates the lifetime of oligomeric assemblies of Dam1 ${ }^{\text {WT }}$ complex on microtubules. By contrast, 'tracer' Dam $1^{\text {OD }}$ complexes were unaffected by the addition of excess un-tagged Dam1 ${ }^{\mathrm{OD}}$ complex. The residence time for 'tracer' Dam1 ${ }^{8 D}$ complexes was $7.0 \pm 0.6 \mathrm{~s}$ (Supplementary Fig. 2d,f), indistinguishable from the residence time in the absence of excess un-tagged Dam1 ${ }^{\mathrm{OD}}$ complexes, $7.0 \pm 0.5 \mathrm{~s}$ (Fig. 1c). Thus, the Dam18D complex does not form stable oligomers on microtubules. Interestingly, the mean residence time of 'tracer' Dam $1^{\mathrm{OD}}$ complexes increased 1.8-fold $(12.5 \pm 1.2 \mathrm{~s})$ in the presence of excess unlabeled Dam $1^{\mathrm{WT}}$ complexes, indicating that the Dam1 ${ }^{\mathrm{OD}}$ complex can associate with the Dam1 WT complex (Supplementary Fig. 2e,f). Because the residence time is only doubled, the OD complex must only associate with one or a few Dam1 $1^{\text {WT }}$ complexes and does not form rings. Finally, by combining gel filtration and velocity sedimentation analyses, we estimated the molecular weight of the Dam1 $1^{\mathrm{WT}}$ and Dam 1 OD complexes to assess oligomerization state in solution. Even at concentrations above $1 \mu \mathrm{M}$, the Dam1 ${ }^{\mathrm{OD}}$ complex is monomeric in solution (Supplementary Fig. 3), while the Dam1 ${ }^{\mathrm{WT}}$ complex exists primarily as a dimer, as reported previously ${ }^{12,16}$.

Unlike the Dam $1^{\mathrm{WT}}$ complex, the Dam1 ${ }^{\mathrm{OD}}$ complex does not dimerize in solution or homo-oligomerize on microtubules. However, the microtubule-binding site of the Dam ${ }^{\mathrm{OD}}$ complex appears intact at the single-molecule level and behaves similar to a single Dam $1^{\mathrm{WT}}$ complex. Therefore, the interfaces that mediate microtubule-binding and oligomerization are contained in structurally distinct modules within the Dam1 complex. 
a
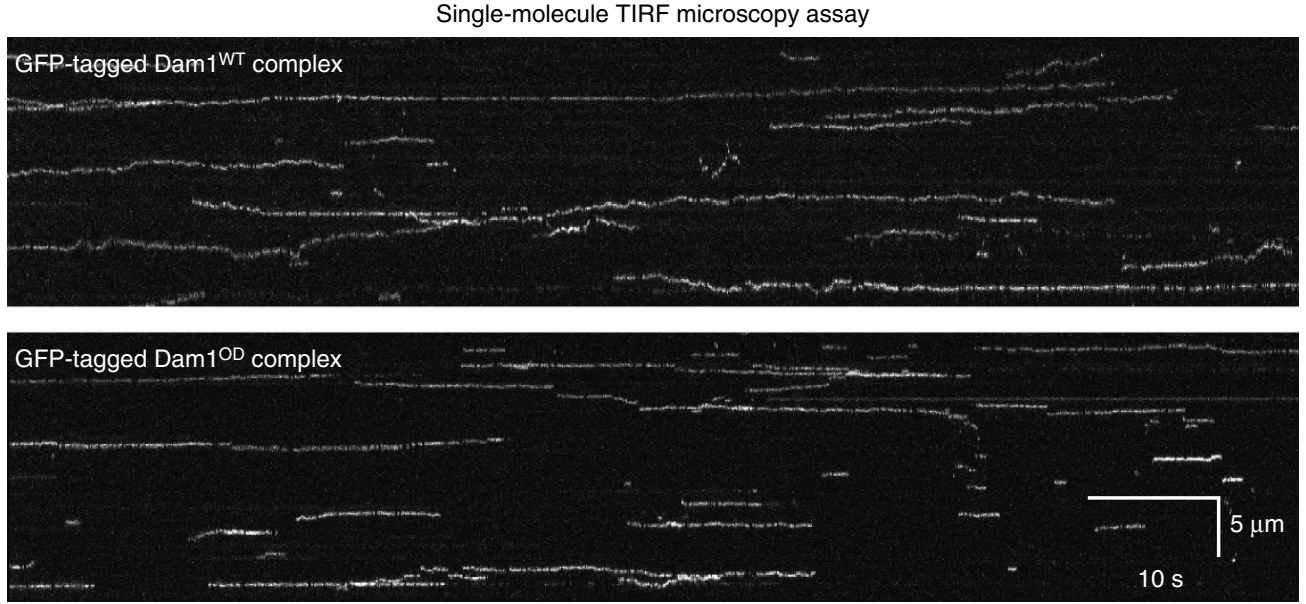

b

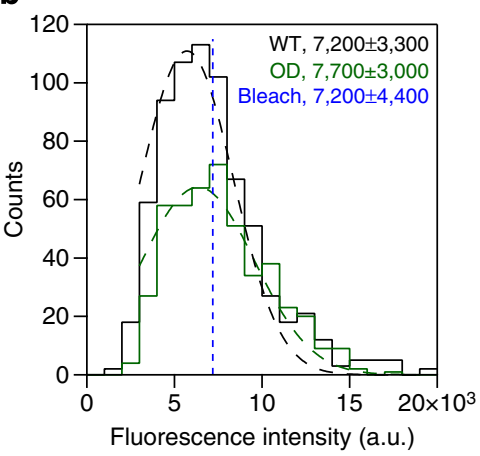

c

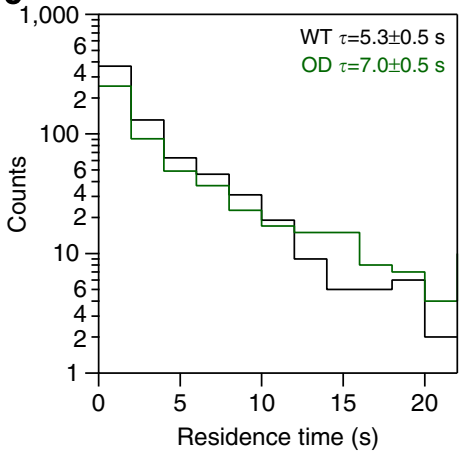

d

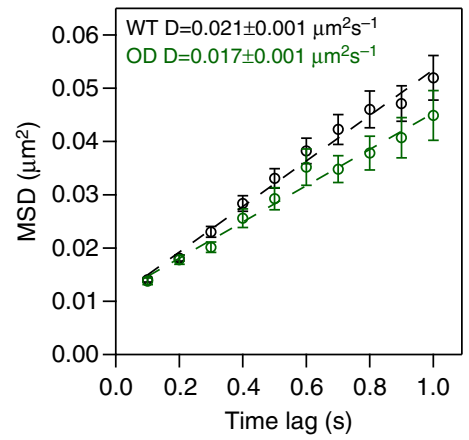

Figure 1 | The oligomerization-deficient Dam1 complex retains an intact microtubule-binding site. (a) Kymographs show single molecules of GFP-tagged wild-type (top) and oligomerization-deficient (bottom) Dam1 complex as they bound to and diffused along taxol-stabilized microtubules. Scale bar applies to both kymographs; image brightness and contrast were adjusted identically. (b) Histograms of GFP brightness intensity for single molecules of wild-type (WT, black trace, $n=717)$ and oligomerization-deficient $(O D$, green trace, $n=472)$ Dam1 complex on microtubules. Dashed-blue line indicates the average height of single-step photobleaches under identical imaging conditions $(n=295)$. Brightness values are reported as mean $\pm \mathrm{s}$.d. (c) Residence time distributions for individual Dam1 ${ }^{\mathrm{WT}}(n=717)$ and $\mathrm{Dam}_{1} \mathrm{OD}(n=564)$ complexes on microtubules. Mean residence time, $\tau \pm$ error of the mean was estimated by bootstrapping analysis (see Methods for additional details). (d) Mean-squared displacement (MSD) versus time lag for single particles (WT, $n=654 ; O D, n=506$ ) represented in $\mathbf{c}$ and $\mathbf{d}$. Error bars on each point indicate s.e.m. Dashed lines indicate weighted linear fits to the data, yielding the diffusion constants, $\mathrm{D} \pm$ s.d. of the fit.

These modules can be separated to measure how oligomerization contributes to kinetochore-microtubule attachment in vitro and in vivo.

Oligomerization is required for the strongest attachments. An optical trap-based rupture force assay ${ }^{12,17}$ was used to assess the strength of microtubule attachments mediated by the WT Dam1 complex and by the oligomerization-deficient complex (Fig. 2a,b). Daml complexes were loaded onto polystyrene beads at relatively high surface density to promote the formation of oligomers, such that up to $\sim 80$ individual complexes could simultaneously interact with the microtubule tip ${ }^{18}$. Beads coated with Dam 1 OD complexes yielded a median rupture force similar to that for beads coated with Dam1 ${ }^{\mathrm{WT}}$ complexes $(4.8$ and $5.2 \mathrm{pN}$, respectively). However, there is an extended 'tail' on the distribution for Dam $1^{\mathrm{WT}}$ complex at very high rupture forces; this tail is absent for the Dam1 ${ }^{\mathrm{D}}$ complex (Fig. 2c, Table 1). The maximum rupture force observed for Dam $1^{\mathrm{WT}}$ complex, $15.2 \mathrm{pN}$, is almost twofold higher than that for the Dam1 ${ }^{\mathrm{OD}}$ complex, $8.3 \mathrm{pN}$. This was not an isolated instance, as $13 \%$ of all ruptures for Dam $1^{\mathrm{WT}}$ complex occurred above the maximum rupture force for the Dam1 ${ }^{\mathrm{OD}}$ complex (Fig. 2c). These findings suggest that oligomerization is required for the formation of strong microtubule attachments.

To further investigate this hypothesis, we performed the rupture force assay in the presence of additional Dam 1 complex added free in solution. By design of the assay, free Dam 1 complex was unable to load directly onto the bead surface, and could only affect attachment strength by oligomerizing with bead-bound Dam1 complex. Relative to Dam 1 WT complex on the beads alone, the addition of $2 \mathrm{nM}$ free Dam $1^{\mathrm{WT}}$ complex significantly increased the proportion of strong attachments (Fig. 2d, Table 1): the median rupture force increased to $6.5 \mathrm{pN}$, and $30 \%$ of all ruptures occurred at high force (above $8.3 \mathrm{pN}$ ). In contrast, the behaviour of Dam $1^{\mathrm{WT}}$ complex on beads was not enhanced by the addition of free Dam $1{ }^{\mathrm{OD}}$ complex (Fig. 2d), even when 10 -fold more was added to compensate for its decreased microtubule decoration seen by TIRF microscopy. Based on our observation that the Dam1 $1^{\mathrm{OD}}$ and Dam1 $1^{\mathrm{WT}}$ complexes can interact directly, we further reasoned that free Dam 1 OD complex might interfere with oligomerization by the Dam1 ${ }^{\mathrm{WT}}$ complex. Indeed, the enhancement in strength afforded by free Dam1 ${ }^{\mathrm{WT}}$ complex was completely negated by simultaneous addition of free Dam1 ${ }^{\mathrm{OD}}$ complex in solution (Fig. 2d). Therefore, we propose that the Dam $1^{\mathrm{OD}}$ complex acts as a competitive inhibitor in the 
a

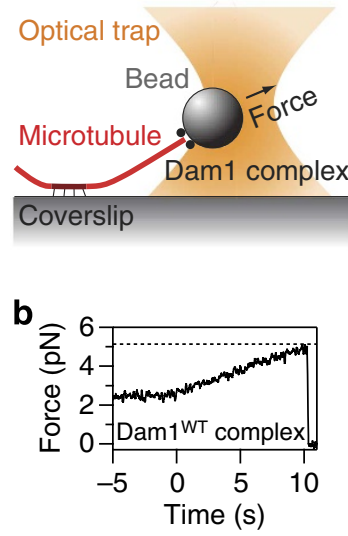

c

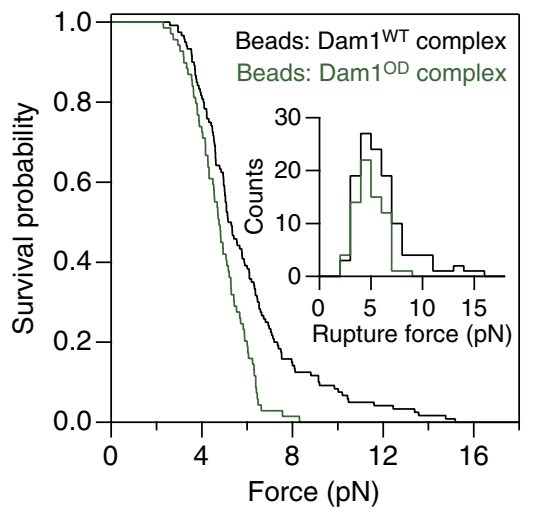

d

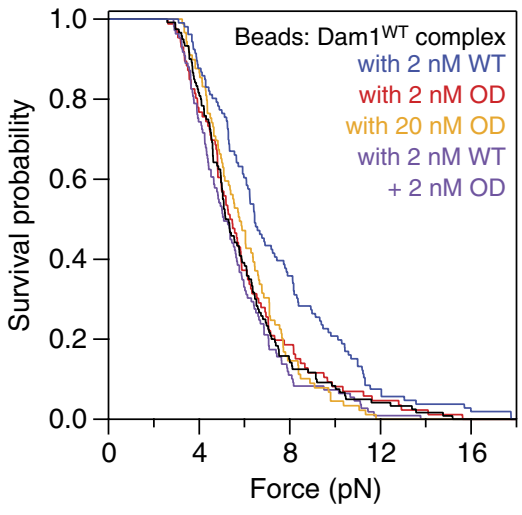

Figure 2 | Oligomerization of the Dam1 complex enhances microtubule attachment strength. (a) Diagram of the optical trap assay. (b) Example trace of applied force versus time from the rupture force assay. Beads are subjected to a $\sim 2.5$-pN test force prior to initiation of the force ramp $\left(\sim 0.25 \mathrm{pN} \mathrm{s}^{-1}\right)$, which begins at $t=0 \mathrm{~s}$. Dashed line indicates the rupture force from this event. (c) Survival versus force curves for beads coated with wild-type (WT, black trace, $n=120$ ) or oligomerization-deficient (OD, green trace, $n=69)$ Dam1 complexes coupled to microtubule tips. Inset: same data represented in survival curves but re-plotted as rupture force histograms. (d) Survival versus force curves for beads coated with Dam 1 WT complex alone (black trace, reproduced from c) or in the presence of additional Dam1 complex free in solution: with $2 \mathrm{nM}$ Dam1 WT complex, blue, $n=106$; with $2 \mathrm{nM} \mathrm{Dam} 1^{\mathrm{OD}}$ complex, red, $n=86$; with $20 \mathrm{nM}$ Dam1 ${ }^{\mathrm{OD}}$ complex, orange, $n=89$; with $2 \mathrm{nM}$ Dam1 ${ }^{\mathrm{WT}}$ and $2 \mathrm{nM}$ Dam1 ${ }^{\mathrm{OD}}$ complex, purple, $n=109$.

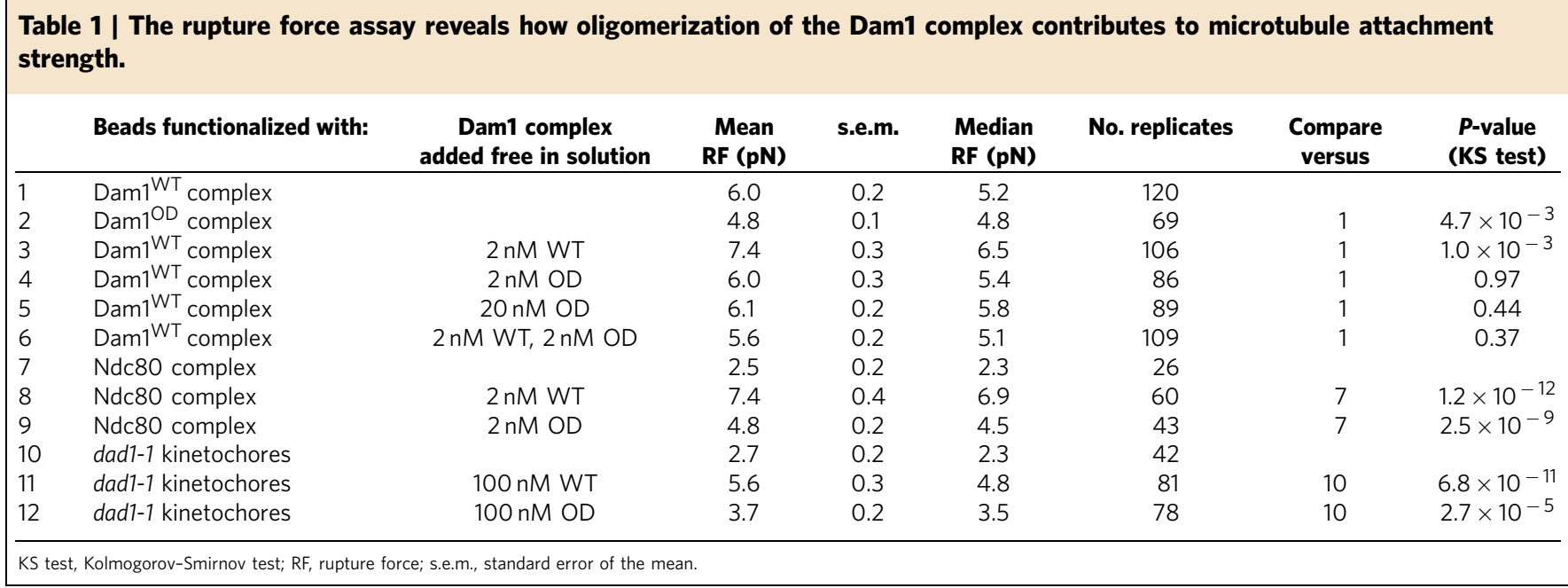

rupture force assay by 'capping' Dam 1 WT oligomers. Our results demonstrate that oligomers of Dam1 complex are necessary to sustain microtubule attachments against high forces.

Oligomerization is not required to bind kinetochores. In vivo, the Ndc80 to complex is required for the Dam1 complex to associate with kinetochores, which occurs after initial kinetochore capture on the lateral face of spindle microtubules ${ }^{2,3,13}$. The Dam1 complex promotes kinetochore-microtubule attachment strength and is required for maturation of these attachments from lateral to end-on ${ }^{1,2}$. Using the optical trap-based rupture force assay, we tested if the Dam1 complex must oligomerize to bind the Ndc80 complex and strengthen its attachment to microtubules. As previously reported ${ }^{12}$, the $\mathrm{Ndc} 80$ complex formed weak microtubule attachments (mean rupture force: $2.5 \pm 0.2 \mathrm{pN}$, error indicates s.e.m.) that were greatly enhanced by the addition of the Dam1 $1^{\mathrm{WT}}$ complex $(7.4 \pm 0.4 \mathrm{pN})$ free in solution (Fig. 3a, Table 1). The Dam 1 OD complex also strengthened Ndc80-based attachments $(4.8 \pm 0.2 \mathrm{pN})$, albeit to a lesser extent than WT (Fig. 3a, Table 1). We further asked if the Dam $1^{\mathrm{OD}}$ complex could bind to native kinetochore particles isolated from budding yeast, and strengthen their attachments to microtubules in vitro. To inactivate endogenous Dam 1 complex, kinetochore particles were purified from cells carrying the temperature-sensitive dad1-1 mutation after being shifted to the restrictive temperature. Consistent with previous work ${ }^{10,11}$, WT kinetochore particles yielded a mean rupture force of $9.7 \pm 1.0 \mathrm{pN}$, while dad1-1 kinetochores were much weaker at $2.7 \pm 0.2 \mathrm{pN}$ (Fig. 3b, Table 1). The mean rupture force of dad1-1 kinetochores was partially rescued by the addition of Dam $1^{\mathrm{WT}}$ complex to $5.6 \pm 0.3 \mathrm{pN}$ (Fig. 3b, Table 1). The Dam $1{ }^{\mathrm{OD}}$ complex was less effective but still partially rescued attachment strength to $3.7 \pm 0.2 \mathrm{pN}$ (Fig. 3b, Table 1). Thus, oligomerization is not strictly required for the Dam 1 complex to transmit load to the kinetochore through the $\mathrm{Ndc} 80$ complex.

Robust coupling against tension requires oligomerization. At the kinetochore, the Dam1 complex enhances the strength and 
a

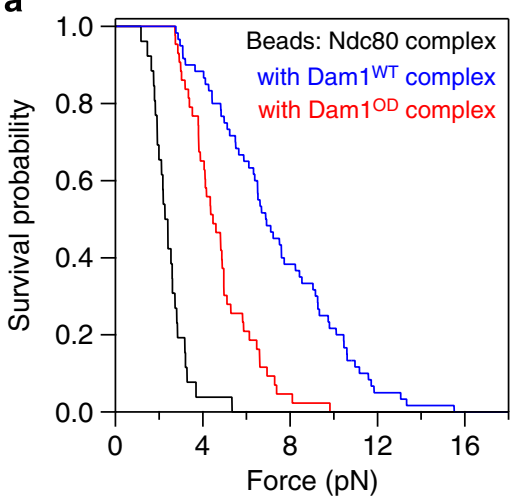

b

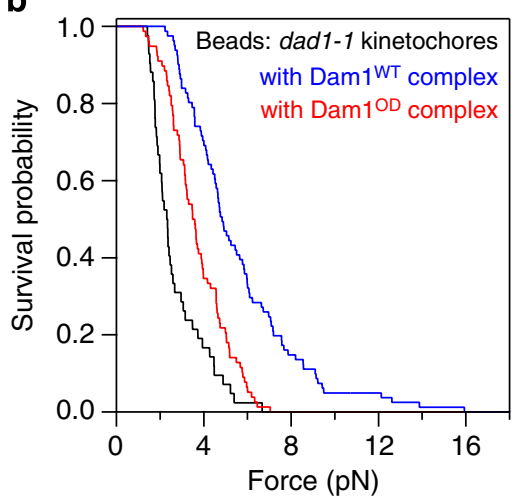

Figure 3 | The Dam1 complex loads onto kinetochores in the absence of oligomerization. (a) Survival versus force curves for beads coated with Ndc80 complex alone (black trace, $n=26)$ or in the presence of free wild-type (WT, blue trace, $n=60)$ or oligomerization-deficient $(O D$, red trace, $n=43$ ) Dam1 complex in solution. (b) As in $\mathbf{a}$, using beads instead coated with purified kinetochore particles lacking functional endogenous Dam1 complex (dad1-1 kinetochores). Beads were assayed alone (black trace, $n=42$ ), and in the presence of purified, recombinant Dam1 WT (blue trace, $n=81$ ) or $\mathrm{Dam}^{\mathrm{OD}}$ (red trace, $n=78$ ) complex added free in solution.

processivity of microtubule linkages ${ }^{12,13}$. Stable kinetochoremicrotubule coupling is crucial when chromosomes become bioriented by the mitotic spindle, as tension is applied across sister kinetochore pairs. It has long been hypothesized that oligomerization is required for the Dam1 complex to support robust coupling to dynamic microtubule ends against applied mechanical loads 4,5 . To test this hypothesis, we programmed the laser trap to apply constant force against Dam1 complexes as they coupled beads to dynamic microtubule tips $s^{9,19}$. When linked directly to beads, both $\mathrm{Dam} 1^{\mathrm{WT}}$ and $\mathrm{Dam} 1^{\mathrm{OD}}$ complexes were able to maintain attachments for assembling microtubule tips over the course of several minutes $(9.9 \pm 1.9 \mathrm{~min}$ and $2.8 \pm 0.3 \mathrm{~min}$, respectively; error is estimated from counting statistics) against a constant load of $2.4 \mathrm{pN}$ (Fig. 4a). During this time, the Dam1 $1^{\mathrm{WT}}$ and $\mathrm{Dam} 1^{\mathrm{OD}}$ complexes had similar effects on microtubule dynamics. Microtubule tips exhibited similar average rates of growth and catastrophe, the transition from assembly to disassembly (Fig. $4 \mathrm{~b}, \mathrm{c}$ ). Following catastrophe events, beads coated with $\mathrm{Dam} 1^{\mathrm{WT}}$ complex coupled to disassembling microtubules for $1.0 \pm 0.3 \mathrm{~min}$ against the applied force. Furthermore, Dam $1^{\mathrm{WT}}$ complex slowed the rate of microtubule disassembly and promoted rescue (the transition from shortening to growth), as previously reported ${ }^{9}$ (Fig. 4b,c). By contrast, beads decorated with $\mathrm{Dam1}{ }^{\mathrm{OD}}$ complex failed to couple robustly for disassembling tips against force, as evidenced by their detachment within $0.05 \pm 0.01 \mathrm{~min}(3 \mathrm{~s})$ on average (Fig. 4a). Dam1 ${ }^{\mathrm{OD}}$ complex also regulated disassembly speed less effectively, and we were unable to observe any rescue events due to the short lifetime of these attachments (Fig. 4b,c). Importantly, the $\mathrm{Dam1} 1^{\mathrm{OD}}$ complex was impaired in disassembly-driven motility only when acting against an applied force. In the absence of force, every bead tested $(n=15)$ underwent processive tracking with a disassembling microtubule end. Most beads (13 of 15) tracked disassembly all the way to the stabilized microtubule seed, while only two events ended in detachment, yielding a mean lifetime of $\sim 5 \mathrm{~min}$ during disassembly in the absence of force. Thus, the oligomerization-deficient Dam1 complex is strikingly defective in coupling to and regulating the dynamics of disassembling microtubule tips under load.

Oligomerization is important for biorientation in vivo. Based on our in vitro results, we predicted that the oligomerizationdeficient Dam1 complex should localize to kinetochores and bind microtubules in vivo, but fail to mediate kinetochore-microtubule attachments against the forces experienced during biorientation. Since deletion of HSK3 is lethal in budding yeast ${ }^{20,21}$, we targeted Hsk3 with an inducible degron system ${ }^{22}$ to transiently generate the Dam $1^{\mathrm{OD}}$ complex in vivo (Supplementary Fig. 4a,b). Degron induction caused cells to arrest with large buds, indicating mitotic arrest and suggesting an essential role for Hsk3 during mitosis. Localization of the two structural modules of the Dam1 complex was tracked in Hsk3-depleted cells using Dad1-GFP (in the microtubule-binding module) and Ask1yellow fluorescent protein (YFP) (in the oligomerization module). Consistent with the formation of the $\mathrm{Dam} 1 \mathrm{OD}$ complex in vivo, depletion of Hsk3 decoupled the localization of these two modules. Dad1-GFP retained localization to kinetochores and the mitotic spindle similar to control cells, while Ask1-YFP became delocalized (Fig. 5a,b and Supplementary Fig. 4c,d). To verify that this is a specific outcome of depleting Hsk3, rather than a generic result of disrupting the Dam1 complex, we targeted the degron system to the Dam1 protein (Supplementary Fig. 5a,b). Depletion of Dam1 caused both Dad1-GFP and Ask1-YFP to delocalize (Fig. $5 c$ and Supplementary Fig. 5c,d). These findings suggest that the Hsk3-degron system generates a stable Dam $1^{\mathrm{OD}}$ complex in vivo and does not simply disrupt the entire Dam1 complex.

To ask if oligomerization is required for normal kinetochoremicrotubule attachment, cells were depleted of Hsk3 and imaged as they entered mitosis. Upon separation of the duplicated spindle pole bodies, kinetochores (marked by Nuf2-GFP) in control cells aligned symmetrically between the two spindle poles, while in Hsk3-depleted cells, kinetochores often aligned asymmetrically towards one pole (Fig. 6a-c). Hsk3-depleted cells additionally exhibited abnormal spindle lengths (for example, Fig. 6b shows spindle hyper-extension), consistent with the high incidence of spindle breakage observed in an extended degron-induced arrest (Supplementary Fig. 6). Asymmetric kinetochore clustering towards one pole is inconsistent with a lack of kinetochoremicrotubule attachment, which causes unclustering of kinetochores in the nucleus ${ }^{23-26}$. Instead, this asymmetric kinetochore clustering phenotype suggests a defect in sister kinetochore biorientation ${ }^{3,27}$. To quantify asymmetry in the kinetochore distribution, we developed a 'kinetochore intensity ratio' metric. Nuf2-GFP fluorescence intensity was integrated across the two halves of each spindle (Fig. 6a,b), and a ratio was taken of the brighter side over the dimmer side (and is thus always $\geq 1$ ). 
a

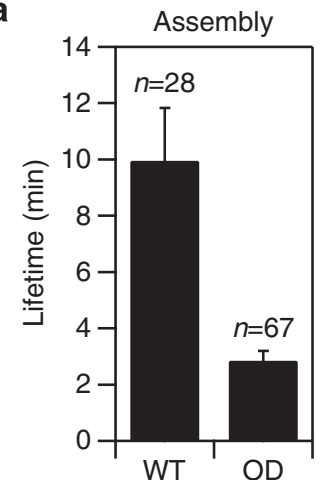

b
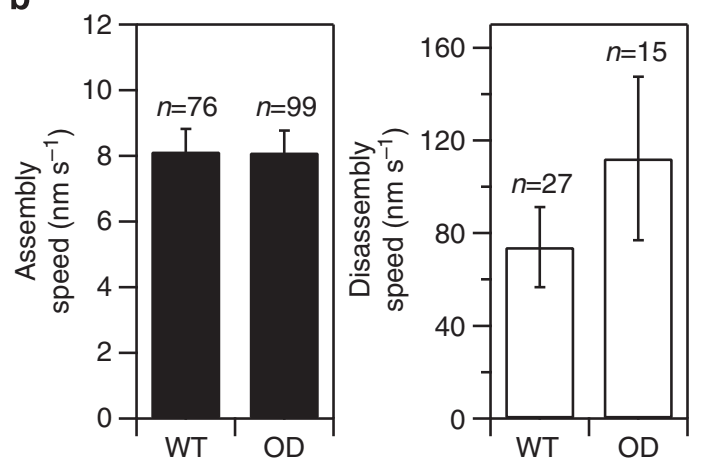

C
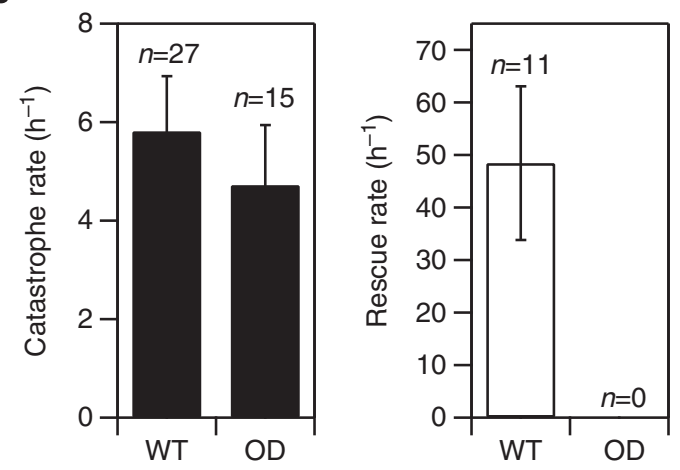

Figure 4 | The oligomerization-deficient Dam1 complex fails to track with disassembling microtubule tips against applied load. Beads coated with wild-type (WT) or oligomerization-deficient (OD) Dam1 complex were tracked while they coupled to assembling (left plots) and disassembling (right plots) microtubule tips under a constant force of $\sim 2.4 \mathrm{pN}$ applied in the direction of microtubule assembly. (a) Mean attachment lifetime for beads coupled to dynamic microtubule tips under constant load. (b) Speeds of microtubule tip assembly and disassembly while coupled to beads under constant force. In our assay conditions, the intrinsic rates of microtubule assembly and disassembly are $\sim 6 \mathrm{~nm} \mathrm{~s}^{-1}$ and $\sim 230 \mathrm{nms}^{-1}$, respectively 9,34 . (c) Switch rates for microtubule tip state: from assembly to disassembly (catastrophe), and from disassembly to assembly (rescue). For microtubules alone, catastrophes occur at a frequency of $\sim 10 \mathrm{~h}^{-1}$ (refs 9,45), and rescues at $\sim 2 \mathrm{~h}^{-1}$ (ref. 34). In a-c, error bars indicate s.e.m.

For control cells, this ratio was narrowly distributed around the average of $1.1 \pm 0.02$ (s.e.m.), indicating accurate bipolar segregation of kinetochores with high fidelity (Fig. 6d,e). By contrast, kinetochore intensity ratios for Hsk3-depleted cells varied widely (from 1.0 to 14 ) and averaged $3.3 \pm 0.6$, reflecting a strong bias towards monopolar alignment that was evident across all observed spindle lengths (Fig. 6d,e). Therefore, the
Microtubule-binding module

Oligomerization module

a
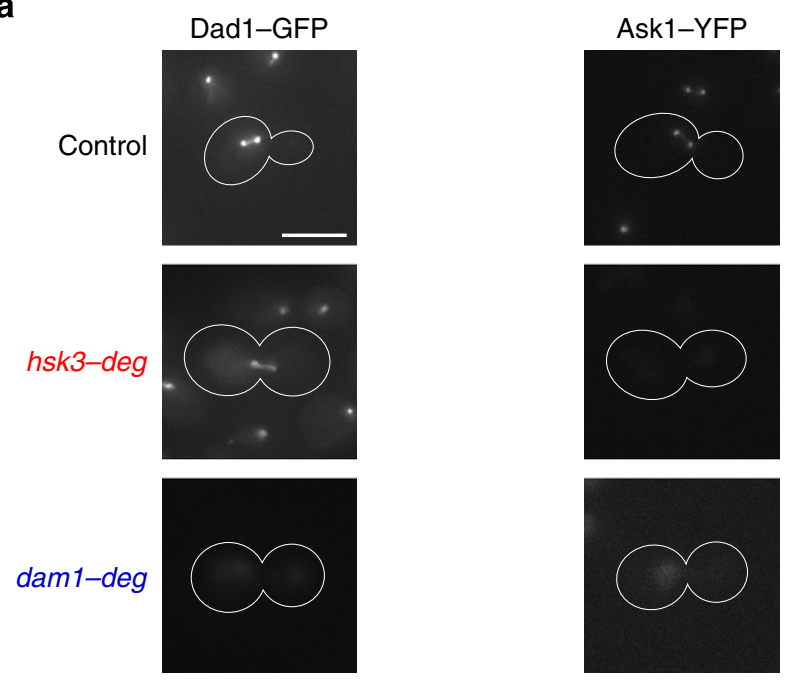

b
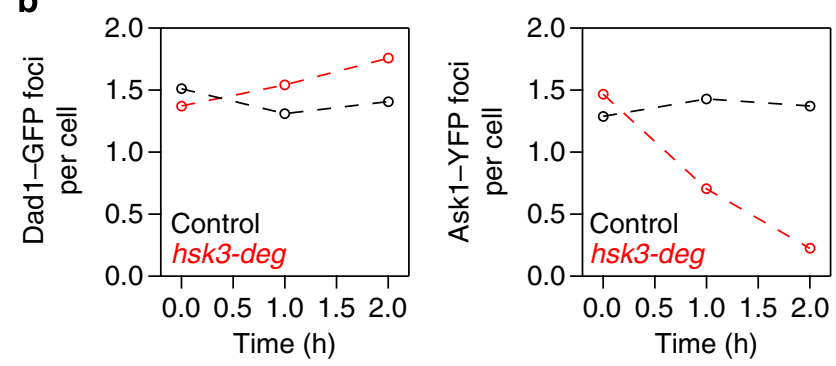

C
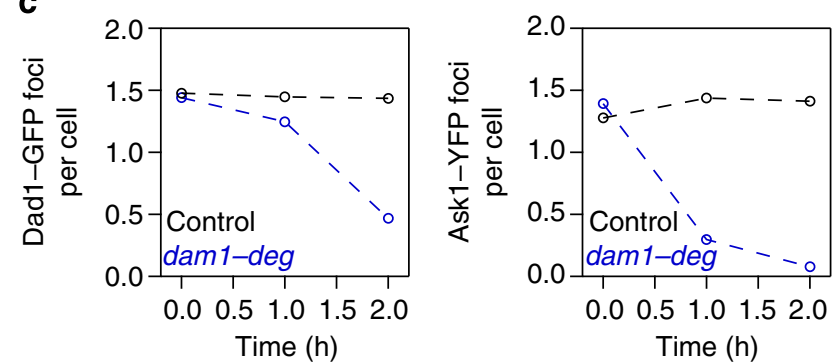

Figure 5 | Depletion of Hsk3 causes formation of the oligomerizationdeficient Dam1 complex in vivo. (a) Representative images of Dad1-GFP and Ask1-YFP in mitotic control (top), hsk3-degron (middle) and dam1degron (bottom) cells at $2 \mathrm{~h}$ after addition of auxin. Scale bar is $5 \mu \mathrm{m}$ and applies to all images. Localization of Dad1-GFP (b) and Ask1-YFP (c) was quantified by automated spot detection and is reported as the average number of detected foci per cell. For both Dad1-GFP and Ask1-YFP, asynchronously growing cells have $\sim 1.4$ foci per cell on an average, indicating a mix of mitotic ( 2 foci) and non-mitotic ( 1 focus) cells. Arrest in mitosis with kinetochore localization causes the average to approach 2.0 foci per cell; delocalization is indicated by a trend towards 0 foci per cell. See Supplementary Figs 3 and 4 for the number of cells counted in each condition.

equal partitioning of kinetochores during mitosis requires oligomerization of the Dam1 complex.

To directly test for a defect in sister kinetochore biorientation, we visualized individual sister centromere pairs in Hsk3-depleted cells. Consistent with the asymmetric distribution of kinetochores, the majority of Hsk3-depleted cells (83\%) had a monopolar CEN3 pair, while only $17 \%$ contained a CEN3 pair 
a

Control (Dam1 ${ }^{\text {WT }}$ complex)
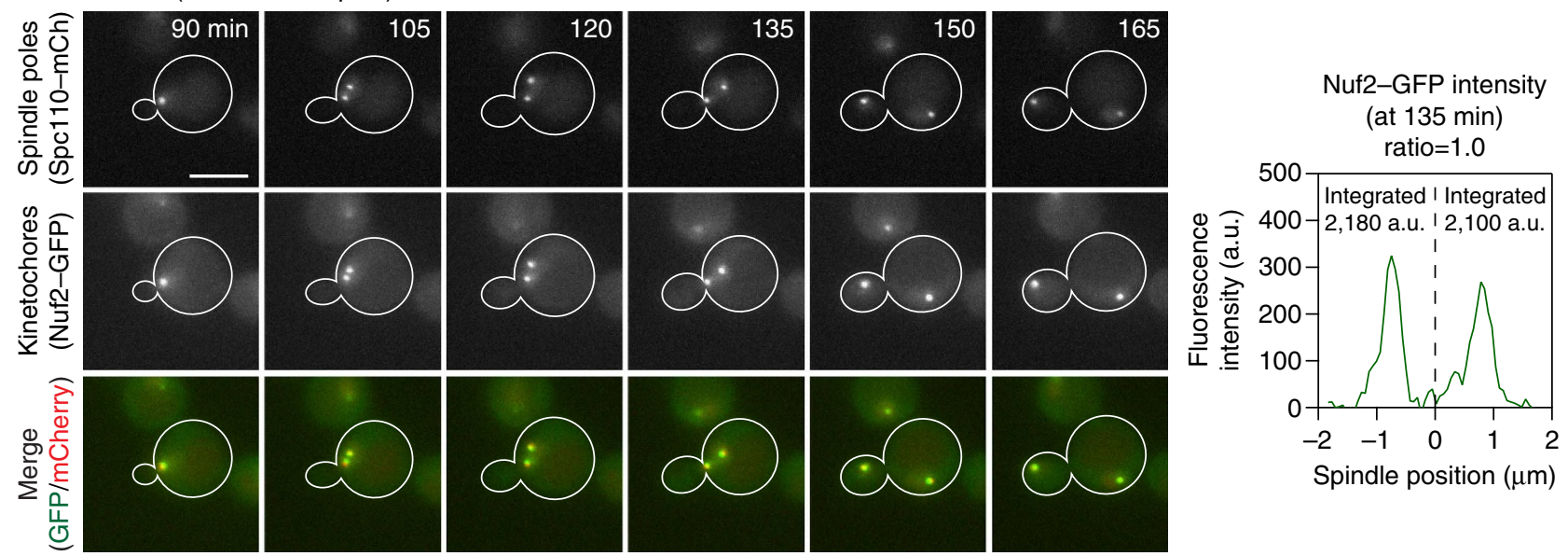

\section{b}

hsk3-degron (Dam1 ${ }^{\mathrm{OD}}$ complex)
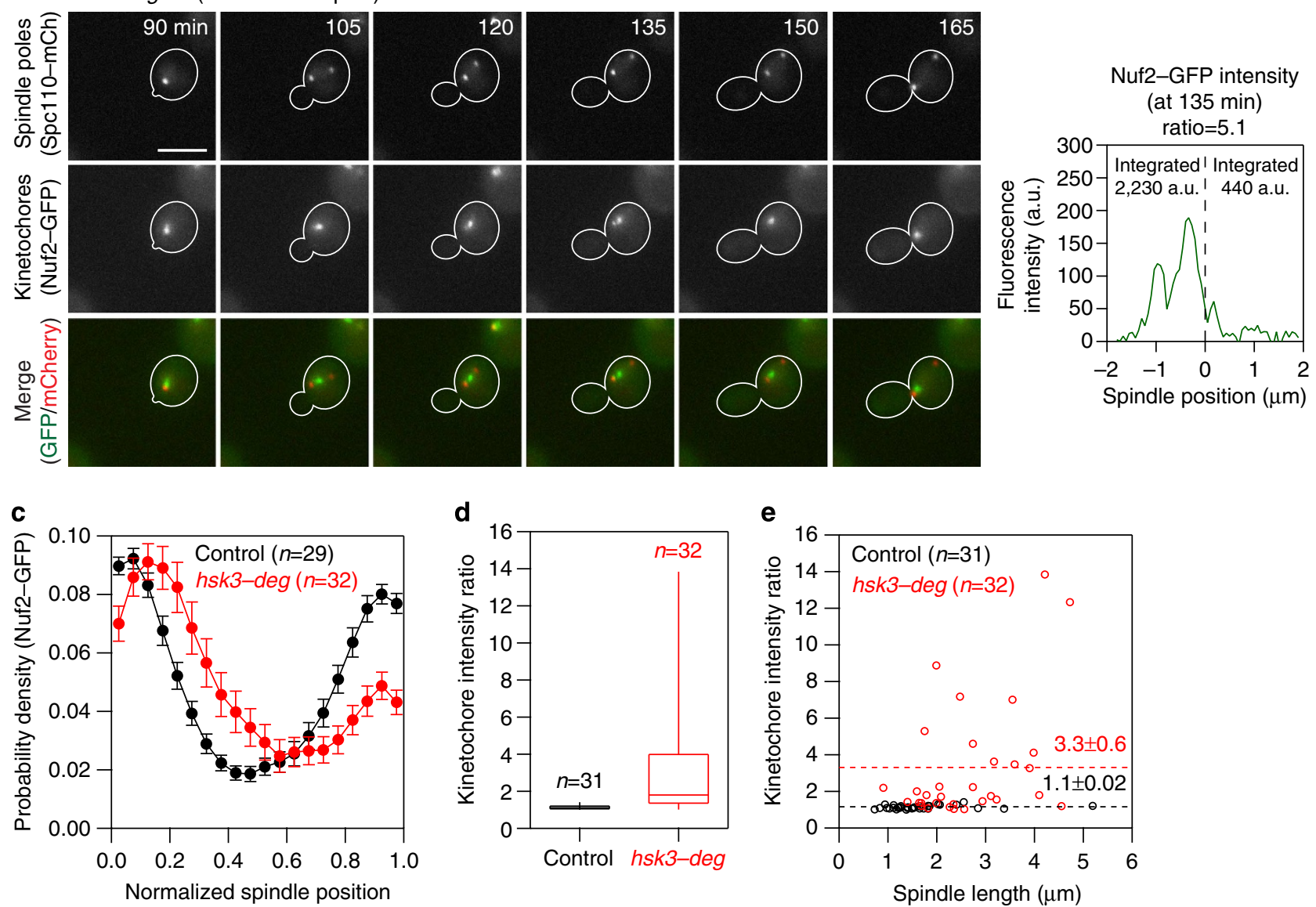

Figure 6 | Equal bipolar distribution of kinetochores requires oligomerization of the Dam1 complex. Time-lapse images of spindle poles (Spc110mCherry, top row), kinetochores (Nuf2-GFP, middle row) and merged channels (bottom row) in control (a) and hsk3-degron (b) cells. Cells were imaged immediately following release from $\alpha$-factor (G1) arrest; time stamps show time after release. Scale bars, $5 \mu \mathrm{m}$. At right in both $\mathbf{a}$ and $\mathbf{b}$ are spindle profiles of Nuf2-GFP intensity from corresponding images taken at $135 \mathrm{~min}$ after release. The integrated intensity in each half-spindle is printed on the plot. (c) Average spindle intensity profiles of Nuf2-GFP in control (black curve) and hsk3-degron (red curve) cells. Markers indicate average \pm s.e.m. (d) Box and whisker plots show the distribution of kinetochore intensity ratios measured for control and hsk3-degron cells. Whiskers show the full range of the distribution, while the box shows the 75th, 50th and 25th percentiles. (e) Plot of kinetochore intensity ratio versus spindle length shows that asymmetric kinetochore alignment in hsk3-degron cells is independent of spindle length. See Methods for additional information about the measurements presented in this figure. 
in a bipolar state (Fig. 7). Of the bipolar CEN3 pairs, 31\% collapsed to monopolar attachments within $30 \mathrm{~min}$ of observation (Fig. 7c and Supplementary Fig. 7). These events provide direct evidence for microtubule attachment failure by one kinetochore of the sister pair. In control cells, weak or erroneous attachments were rarely observed. Among 112 control cells, only
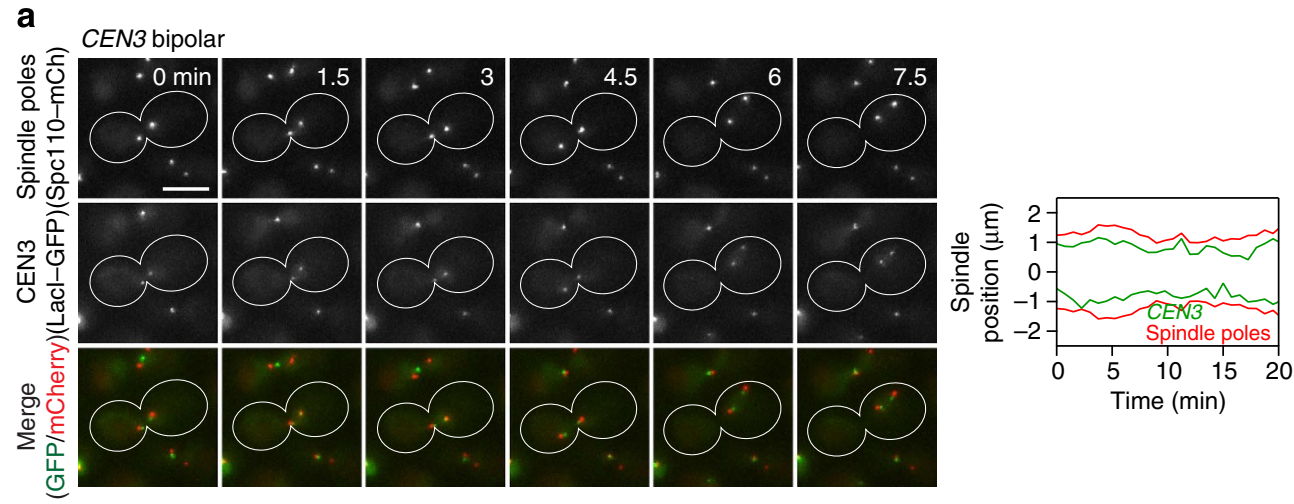

b

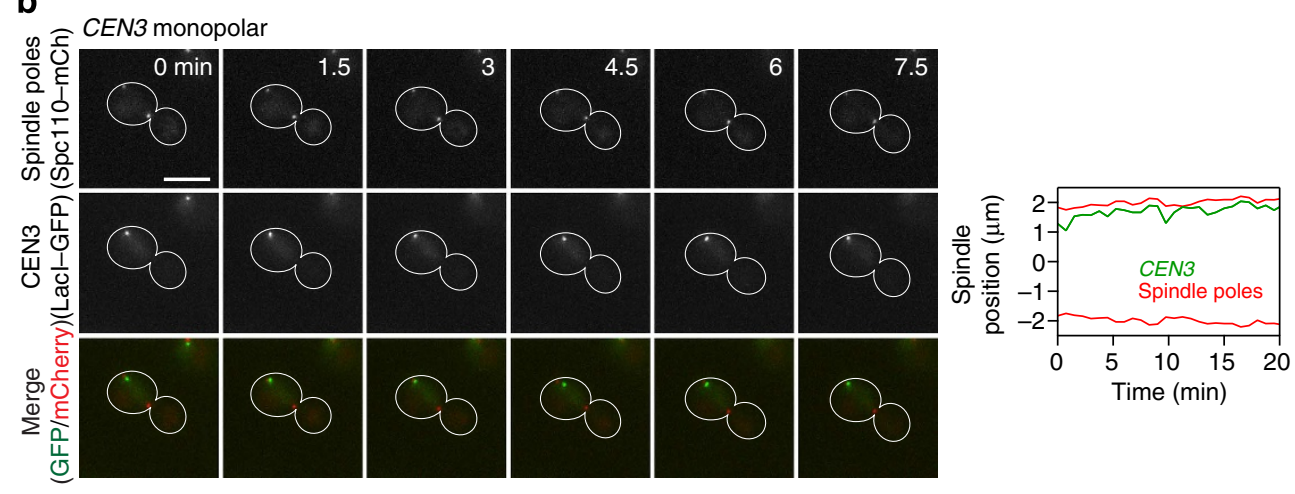

C
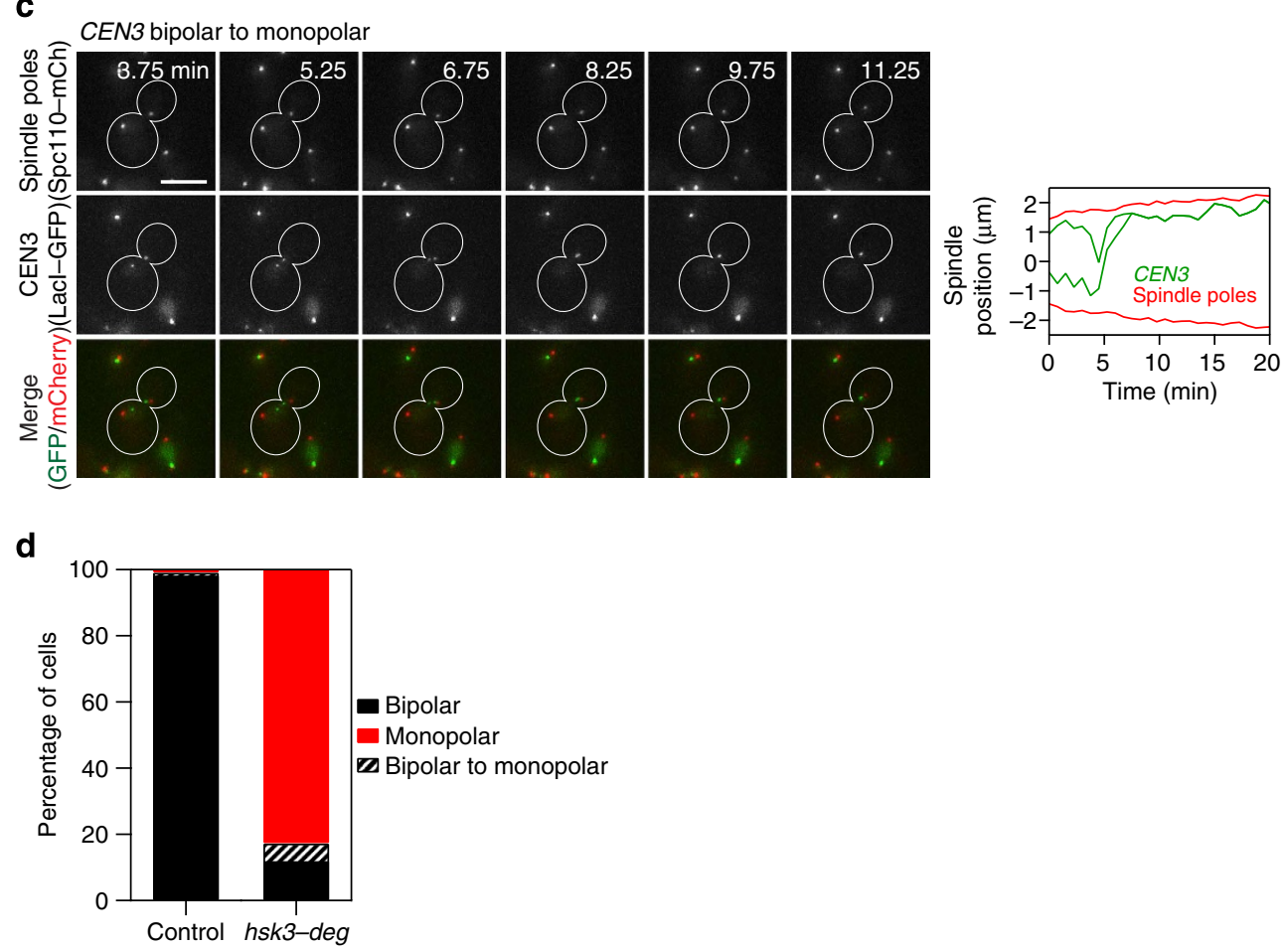

Figure 7 | The oligomerization-deficient Dam1 complex fails to support sister kinetochore biorientation. Representative time-lapse images of hsk3-degron cells show the three classes of CEN3 behaviour: bioriented (a), monopolar (b) and biorientation failure (c). GFP and mCherry channels are shown separately and merged as in Fig. 6 . Scale bars, $5 \mu \mathrm{m}$; contrast was adjusted for visual clarity. Time stamps indicate elapsed time from the start of imaging. Shown to the right in a-c are the plots of position versus time for spindle poles (red) and CEN3 spots (green) from the corresponding time-lapse series. (d) Bar graph shows the percentage of cells in each CEN3 class for control $(n=121)$ and hsk3-degron $(n=168)$ cells. 
one (1\%) contained a monopolar CEN3 spot, and the remaining (99\%) bioriented CEN3 (Fig. 7d). Furthermore, nearly all cells (99\%) with CEN3 biorientation maintained stable separation of the sister pair over the entire imaging period (Fig. 7d). Therefore, the kinetochore biorientation defect in Hsk3-depleted cells directly supports predictions based on our in vitro experiments. Taken altogether, our findings suggest that oligomerization of the Daml complex is required for stable coupling of kinetochores to dynamic microtubule ends under tension in vivo.

\section{Discussion}

The Dam1 complex spontaneously assembles into oligomers in vitro, and forms rings that encircle microtubules. The functional consequences of oligomerization have remained unclear. One possibility is that oligomerization merely increases the avidity by allowing multiple Dam 1 complexes to simultaneously contact the microtubule. Another non-mutually exclusive possibility is that oligomerization forms a structure with a specialized interaction with microtubule ends. For example, in its oligomeric ring conformation, the Daml complex is proposed to hook onto curled protofilaments at disassembling microtubule ends, thereby harnessing mechanical energy from the 'conformational wave' of microtubule disassembly ${ }^{7,28-30}$. However, oligomerization of the Dam1 complex has not been shown to be required for its functions either in forming direct attachments to dynamic microtubule ends in vitro, or in mediating these linkages at kinetochores in vivo.

We addressed this question by using a version of the Dam1 complex that does not form oligomeric rings in vitro ${ }^{14}$. Here we show that this version of the Dam1 complex retains WT microtubule-binding behaviour at the level of single complexes, but lacks the ability to assemble into oligomers of any size, and is thus 'Oligomerization-Deficient' (Dam $1^{\mathrm{OD}}$ complex). In our optical trap assays, we found that oligomerization is required in vitro for the Daml complex to form microtubule attachments that are robust against applied mechanical loads. Due to the design of the assay, this function must be independent of binding avidity. Dam1 complexes were linked to the surface of polystyrene beads such that up to $\sim 80 \mathrm{Dam} 1$ complexes could simultaneously reach a microtubule end. This arrangement on the bead surface artificially imposes avidity; however, avidity alone is insufficient to rescue the defect of $\mathrm{Dam1}{ }^{\mathrm{OD}}$ complex in coupling to microtubule ends under tension. Therefore, oligomerization of the Daml complex contributes to the strength and processivity of microtubule attachments in a manner that is independent of binding avidity. Our findings support the possibility that oligomerization allows the Dam1 complex to form a ring that helps prevent its detachment from a flared microtubule end. We propose that this conformation is required at the kinetochoremicrotubule interface in vivo, especially when kinetochores must sustain these linkages under tension during mitosis.

We tested this hypothesis in vivo using an Hsk3-degron system to generate the oligomerization-deficient Dam1 complex in cells. We found that depletion of Hsk3 releases the oligomerization module of the Dam1 complex from its microtubule-binding module, indicating successful formation of the Dam $1^{\mathrm{OD}}$ complex in vivo. In Hsk3-depleted mitotic cells, bipolar centromere pairs collapsed to monopolar attachments. This microtubule attachment failure at one kinetochore of the sister pair can be explained directly by our in vitro results. The $\mathrm{Dam}{ }^{\mathrm{OD}}$ complex is unable to mediate stable coupling to dynamic microtubule ends under tension. Following breakage, sister pairs remained attached in a monopolar state in vivo, consistent with the ability of the Dam1 ${ }^{O D}$ complex to maintain attachments in the absence of tension in vitro. Thus, in the absence of oligomerization, the microtubule-binding ability of the Dam1 complex alone is insufficient to support stable kinetochore-microtubule attachment under tension in vivo.

Our findings additionally suggest that oligomerization of the Dam1 complex can be regulated by controlling Hsk3 levels. Cells entering meiosis specifically suppress HSK3 expression among the genes encoding members of the Dam1 complex, and this regulation appears to promote the proper segregation of chromosomes during meiosis I (ref. 31). We speculate that the regulation of HSK3 might produce the Dam $1{ }^{\mathrm{OD}}$ complex as the biologically relevant form of the Dam1 complex during meiosis I.

Outside of fungal organisms, homologues of the Dam1 complex have not been identified; however, the Ska complex is a proposed functional equivalent in higher eukaryotes, and has been reported to form oligomeric structures in vitro ${ }^{32}$. Moreover, we and others have found that the human Ndc80 complex has evolved the ability to oligomerize ${ }^{33-35}$. Interestingly, the human Ndc80 complex is thought to oligomerize 'longitudinally' on microtubules, binding along individual protofilaments ${ }^{33}$. This form of oligomerization could provide functions distinct from those afforded by 'lateral' oligomerization (binding around the tube, as seen in Dam1 complex rings). The experiments presented here provide a framework to study how oligomerization contributes to kinetochore-microtubule coupling in other systems. Ultimately, we show that oligomerization is an important mechanism by which kinetochore components are coordinated to form a robust microtubule attachment site.

\section{Methods}

Protein expression and purification. All ten components of the $S$. cerevisiae Dam1 complex were co-expressed in Escherichia coli from a polycistronic vector ${ }^{5}$ The complex was affinity-purified using a C-terminal $\mathrm{His}_{6}$ - $^{-}$or FLAG-tag on Spc34 and subjected to gel filtration, as previously described ${ }^{8,9,12}$. The expression vector was also modified to remove the gene encoding Hsk3; purification as carried out for the WT complex then yielded the six-protein oligomerization-deficient Dam1 complex, as previously reported ${ }^{14}$. For TIRF microscopy experiments, the Dam1 complex was tagged by fusion of GFP to the C-terminus of Dad $1^{8}$. The S. cerevisiae $\mathrm{Ndc} 80$ complex was expressed in E. coli, purified using a C-terminal $\mathrm{His}_{6}$-tag, and subjected to gel filtration, as reported previously $12,18,36$.

Native kinetochore particles were purified from asynchronously growing S. cerevisiae cells using Dsn1-His ${ }_{6}$-FLAG (refs 10,11). Cells were grown in yeast peptone dextrose rich (YPD) medium ${ }^{37}$ and extract was prepared by lysing cells in a blender in the presence of dry ice. Lysed cells were resuspended in buffer $\mathrm{H}(\mathrm{BH})$ (25 mM HEPES pH 8.0, $2 \mathrm{mM} \mathrm{MgCl}_{2}, 0.1 \mathrm{mM}$ EDTA, $0.5 \mathrm{mM}$ EGTA, 0.1\% NP-40, $150 \mathrm{mM} \mathrm{KCl}$ and $15 \%$ glycerol) containing protease and phosphatase inhibitors followed by ultracentrifugation at $98,500 \mathrm{~g}$ for $90 \mathrm{~min}$ at $4^{\circ} \mathrm{C}$. Beads conjugated with anti-FLAG antibodies were incubated with extract for $3 \mathrm{~h}$ with constant rotation, followed by three washes with $\mathrm{BH}$ containing protease inhibitors, phosphatase inhibitors and $2 \mathrm{mM}$ dithiothreitol (DTT). Beads were further washed twice with $\mathrm{BH}$-containing protease inhibitors. Associated proteins were eluted from the beads by gentle agitation of beads in elution buffer $\left(0.5 \mathrm{mg} \mathrm{ml}^{-1} 3 \times\right.$ FLAG $^{-1}$ peptide in $\mathrm{BH}$ with protease inhibitors) for $30 \mathrm{~min}$ at room temperature.

Electron microscopy. Sample preparation and imaging were performed essentially as described previously ${ }^{12}$. In brief, taxol-stabilized microtubules $(36 \mathrm{nM}$ polymerized tubulin) were incubated with $20 \mathrm{nM} \mathrm{Dam} 1^{\text {WT }}$ complex or Dam1 ${ }^{\mathrm{OD}}$ complex. A 2- $\mu$ l drop of sample was applied to a negatively charged carbon-coated copper grid (Electron Microscopy Sciences (EMS)). Grids were washed with BRB80 (80 $\mathrm{mM}$ PIPES, $120 \mathrm{mM} \mathrm{K}^{+}, 1 \mathrm{mM} \mathrm{MgCl}_{2}$ and $1 \mathrm{mM} \mathrm{EGTA}, \mathrm{pH}$ 6.9) and then stained with $0.075 \%$ uranyl formate. Images were collected on a Spirit T12 (FEI) at $\times 52,000$ magnification at the specimen level with $1 \mathrm{~s}$ exposure.

TIRF microscopy. Flow chambers were constructed and functionalized as reported previously ${ }^{8,12,18,38}$. Coverglass was adhered to a glass slide using double sticky tape, forming individual flow channels between two adjacent strips of tape. 'Rigour' kinesin was introduced and allowed to adsorb non-specifically to the glass, allowing the immobilization of taxol-stabilized microtubules on the coverslip. Singlemolecule imaging experiments were carried out by incubating 5-40 pM Dam ${ }^{\mathrm{WT}}$ or Dam1 ${ }^{\mathrm{OD}}$ complex with Alexa-647-labelled microtubules. GFP and Alexa-647 fluorescence channels were simultaneously recorded using a custom TIRF imaging system $^{38}$. In 'tracer' assays, GFP-tagged and unlabeled versions of the Dam1 complex were pre-mixed at a 1:100 ratio to a total concentration of $2 \mathrm{nM}$ (Dam1 WT complex) or $20 \mathrm{nM}$ (Dam1 ${ }^{\mathrm{OD}}$ complex) and subsequently incubated with 
microtubules. All TIRF assays were performed in BRB80 containing $1 \mathrm{mg} \mathrm{ml}^{-1}$ $\kappa$-casein, and supplemented with an additional $70 \mathrm{mM} \mathrm{KCl}$ and an oxygen scavenger system $\left(200 \mu \mathrm{g} \mathrm{ml}^{-1}\right.$ glucose oxidase, $35 \mu \mathrm{g} \mathrm{ml}^{-1}$ catalase, $25 \mathrm{mM}$ glucose and $5 \mathrm{mM}$ DTT).

Single-particle tracking and analysis was performed using custom software (available upon request) developed in Labview (National Instruments) and Igor Pro (Wavemetrics) as reported previously ${ }^{8,12,18}$. Average binding densities of Dam $1^{\text {WT }}$ and $\mathrm{Dam} 1^{\mathrm{OD}}$ complexes on microtubules (Supplementary Fig. $2 \mathrm{~b}$ ) were measured using the line scan function in ImageJ (NIH). Photo-bleaching experiments were carried out by non-specifically adsorbing GFP-tagged Daml complexes to the coverglass at a density of $\sim 100$ complexes per field of view $\left(1,150 \mu \mathrm{m}^{2}\right)$ and imaging under conditions identical to those used in single-molecule experiments. Automated analysis of single-step photo-bleach events was performed by fitting a heavy-side step function to records of brightness intensity over time (custom software developed in Labview, available upon request).

Bootstrapping analysis was used to determine mean residence time and to estimate the error of the mean. Each residence time data set was randomly resampled with replacement 1,000 times. For both Dam $1{ }^{\text {WT }}$ and Dam $1{ }^{\mathrm{OD}}$ complexes, the means of the resampled data sets formed normal distributions. Gaussian fits to these distributions yielded estimates of mean residence time, and the Gaussian width was used as an estimate of the error.

Protein size analysis. Size-exclusion chromatography was carried out using a HiLoad Superdex 16/60 prep grade column (GE Healthcare) equilibrated in Dam1 purification buffer $(500 \mathrm{mM} \mathrm{NaCl}, 50 \mathrm{mM}$ phosphate, $\mathrm{pH}$ 6.9). The column was calibrated using BSA, catalase, ferritin and thyroglobulin as protein size standards. For velocity sedimentation analysis, a $90-\mu l$ sample of $2 \mu \mathrm{M}$ Dam1 ${ }^{\mathrm{WT}}$ or $\mathrm{Dam} 1^{\mathrm{OD}}$ complex was loaded onto a $1-\mathrm{ml} 8-32 \%$ sucrose gradient made in Daml purification buffer; BSA, catalase and aldolase were used as protein size standards.

Optical trap-based bead motility assays. Streptavidin-coated $0.44-\mu \mathrm{m}$ polystyrene beads (Spherotech) were functionalized with biotinylated anti-His ${ }_{5}$ antibody (Qiagen) and decorated with Daml complexes via recognition of the $\mathrm{His}_{6}$-tag on the $\mathrm{C}$ terminus of Spc34. Incubation of $11 \mathrm{pM}$ beads and $20 \mathrm{nM}$ Dam1 complex (for $\sim 1 \mathrm{~h}$ at $4{ }^{\circ} \mathrm{C}$ ) yielded a surface density of $\sim 2,000$ complexes per bead. In this arrangement, we estimate that up to $\sim 80 \mathrm{Dam} 1$ complexes can simultaneously reach from the bead surface to the microtubule end ${ }^{18}$. Bead decoration was performed in a total volume of $60 \mu$ incubation buffer (BRB80 containing $8 \mathrm{mg} \mathrm{ml}^{-1}$ BSA and $1 \mathrm{mM}$ DTT). Any remaining Daml complex not bound to the bead surface was removed by pelleting the beads $\left(16,000 \mathrm{~g}\right.$ for $10 \mathrm{~min}$ at $\left.4{ }^{\circ} \mathrm{C}\right)$ and washing with $\sim 200 \mu \mathrm{l}$ incubation buffer. Beads were returned to the original incubation volume by pelleting $\left(16,000 \mathrm{~g}\right.$ for $10 \mathrm{~min}$ at $\left.4{ }^{\circ} \mathrm{C}\right)$ and resuspending in $60 \mu$ incubation buffer. Beads coated with $\mathrm{Ndc} 80$ complex were prepared in the same manner as described for the Dam1 complex. Kinetochore particles were diluted such that the concentration of Dsn1 was $\sim 0.4 \mathrm{ng} \mathrm{ul}^{-1}$, and then incubated with $6 \mathrm{pM}$ beads for $\sim 1 \mathrm{~h}$ at $4^{\circ} \mathrm{C}$, as described previously ${ }^{10,11}$.

Flow chambers were prepared to immobilize GMPCPP-stabilized microtubule seeds on the coverglass, as previously described ${ }^{17,34}$. Beads were introduced into the flow chamber in assay buffer (BRB80 containing $8 \mathrm{mg} \mathrm{ml}^{-1}$ BSA, $1 \mathrm{mg} \mathrm{ml}^{-1}$ $\kappa$-casein, $1 \mathrm{mM}$ DTT, $1 \mathrm{mM} \mathrm{GTP}$ and $1.4 \mathrm{mg} \mathrm{ml}$ tubulin) supplemented with an oxygen scavenging system $\left(250 \mu \mathrm{g} \mathrm{ml}^{-1}\right.$ glucose oxidase, $30 \mathrm{\mu g} \mathrm{ml}^{-1}$ catalase and $30 \mathrm{mM}$ glucose). Dynamic microtubule extensions were assembled by the addition of free tubulin dimers onto the coverglass-bound GMPCPP-stabilized microtubule seeds.

A custom optical trap instrument ${ }^{17}$ was used to capture and manipulate beads, and to apply force to the attachments between the Dam 1 complex and dynamic microtubule ends. Rupture force experiments were performed essentially as described previously ${ }^{10,11,12}$. Beads were initially attached to microtubules and subjected to a 2.4-pN constant 'test' force in the direction of microtubule growth. Beads were required to track microtubule growth over a distance of $\sim 100-500 \mathrm{~nm}$ to ensure end-on attachment. After satisfying the test phase, the applied force was increased at a constant rate of $0.25 \mathrm{pN} \mathrm{s}^{-1}$ until bead detachment. Records of force versus time collected during the experiment were used to determine the rupture force, which was marked as the maximum force sustained by the attachment during each event. Some rupture force assays included FLAG-tagged Dam1 complex added free in solution along with the beads; this free Daml complex was supplied in the assay buffer.

Constant force experiments were also carried out as previously reported ${ }^{9,18,19}$. Briefly, beads were attached to assembling microtubule ends, and $2.4 \mathrm{pN}$ was applied continuously (in the direction of microtubule growth) throughout rounds of microtubule assembly and disassembly. Upon bead detachment, microtubule tip state was determined visually during the experiment and confirmed during subsequent analysis using traces of bead position versus time. Attachment lifetime and microtubule dynamic rates were also measured from bead position traces using custom software (available upon request) developed in Igor Pro.

For experiments performed in the absence of applied force, beads were tracked by differential interference contrast imaging as previously described ${ }^{19}$. Beads were bound to the microtubule lattice, and a secondary laser was used to induce microtubule disassembly by ablation of the plus end, as reported previously ${ }^{12,34}$
Attachment lifetime was measured from video recordings and was defined as the period over which recognizable disassembly-driven motility occurred.

Yeast culture and live-cell imaging. Strains used in this study are listed in Supplementary Table 1 and are derivatives of SBY3 (W303). NUF2-GFP and SPC110-mCherry (ref. 1), pCUP1-GFP12-LacI12 and CEN3::33LacO (ref. 39), TUB1-GFP (ref. 40) and OsTIR1-9myc constructs ${ }^{22}$ were described previously. ASK1-YFP was created as described ${ }^{41}$. HSK3-3V5-IAA7, DAM1-3V5-IAA7 and DAD1-GFP were constructed by PCR-based methods as reported previously ${ }^{42}$. Primer sequences for strain constructions are available upon request. Auxininducible degron alleles were generated by fusing the protein of interest with 3V5-IAA7. Plasmids for 3V5-IAA7 fusion were kindly provided by Dr Leon Chan (UC Berkeley).

Cultures were grown at $30^{\circ} \mathrm{C}$ in YPD to $\sim 30-60$ Klett. The auxin-inducible degron system ${ }^{22}$ was activated by addition of $0.5 \mathrm{mM}$ auxin to the growth medium (YPD + auxin). Cells were mounted on agar pads as described previously ${ }^{43}$ or in a CellAsic (Millipore) microfluidics device and imaged using a DeltaVision system (Applied Precision) fitted with an IX70 inverted fluorescence microscope (Olympus), a Uplan APO $\times 100$ objective (1.35 NA) and a CoolSnap HQ camera (Photometrics).

For localization experiments, asynchronously growing cells were collected for imaging on agar pads at designated timepoints after the addition of auxin. Exposures of $0.3 \mathrm{~s}$ were recorded for Dad1-GFP or Ask1-YFP in each of nine $0.5-\mu \mathrm{m}$ optical sections. Automated spot detection was performed using custom Matlab-based software, Fluorcal ${ }^{44}$, available upon request. Cells were counted visually using differential interference contrast images taken in the same field of view. Spindle morphology was assessed in asynchronously growing cultures after treatment with auxin for $2 \mathrm{~h}$. Tubl-GFP and Spc110-mCherry were imaged as described for Dad1-GFP and Ask1-YFP. Spindles were considered broken if Tub1-GFP fluorescence was not oriented along the spindle axis.

Kinetochore biorientation was imaged in synchronized cells using the CellAsic system. Asynchronous cells $(\sim 30 \mathrm{Klett})$ were loaded into the microfluidics chamber and grown in YPD $+6 \mu \mathrm{M} \alpha$-factor medium for $2 \mathrm{~h}$. Auxin was then introduced by exchanging to YPD $+6 \mu \mathrm{M} \alpha$-factor $+0.5 \mathrm{mM}$ auxin medium for $1 \mathrm{~h}$. Cells were subsequently released from $\alpha$-factor into YPD + auxin medium and imaged in three 1.5- $\mu \mathrm{m}$ optical sections at 7.5-min intervals from the time of release. Nuf2-GFP and Spc110-mCherry were imaged with exposures of $0.1 \mathrm{~s}$ and $0.3 \mathrm{~s}$, respectively. For each cell, a single representative frame in which both spindle poles were in good focus was used to generate a spindle profile. Spindle profiles were generated from maximum intensity projections of the GFP and mCherry channels with the ImageJ line scan tool. Automated analysis was performed on each spindle profile using custom software developed in Igor Pro, available upon request. Spindle poles were located using Gaussian fitting, and the spindle midzone was defined as the midpoint between the two poles. GFP fluorescence intensity was then integrated in each half spindle (as defined by the spindle midzone) to obtain the 'kinetochore intensity' associated with each spindle pole. The kinetochore intensity ratio was then determined as the larger kinetochore intensity divided by the smaller; by definition, this ratio is always $\geq 1$. Average spindle profiles were obtained by normalizing spindle length: the spindle pole associated with the larger kinetochore intensity was defined as position 0 , and the opposite spindle pole as position 1. GFP intensity was binned in increments of 0.05 along the normalized spindle axis and averaged across all measured spindles.

In biorientation experiments, CEN3 was visualized using LacI-GFP bound to a lacO array adjacent to the centromere. LacI-GFP is under control of the $p C U P 1$ promoter and imaged using uninduced conditions, with media made from yeast nitrogen base lacking copper (ForMedium). Auxin was added to asynchronous cultures, and live cells were imaged on agar pads at 45 -s intervals for up to $30 \mathrm{~min}$. LacI-GFP and Spc110-mCherry were imaged with $0.3 \mathrm{~s}$ exposures in nine $0.5-\mu \mathrm{m}$ optical sections, as described above for Dad1-GFP. Biorientation was scored manually after the experiment for all cells in which both spindle poles remained in focus throughout the observation period. Position versus time traces were obtained from four-dimensional automated spot tracking applied to the mCherry and GFP channels using Imaris (Bitplane).

\section{References}

1. Shimogawa, M. M. et al. Mps1 phosphorylation of Dam1 couples kinetochores to microtubule plus ends at metaphase. Curr. Biol. 16, 1489-1501 (2006).

2. Tanaka, K. et al. Molecular mechanisms of kinetochore capture by spindle microtubules. Nature 434, 987-994 (2005).

3. Janke, C., Ortiz, J., Tanaka, T. U., Lechner, J. \& Schiebel, E. Four new subunits of the Dam1-Duo1 complex reveal novel functions in sister kinetochore biorientation. EMBO J. 21, 181-193 (2002).

4. Westermann, S. et al. Formation of a dynamic kinetochore- microtubule interface through assembly of the Daml ring complex. Mol. Cell 17, 277-290 (2005).

5. Miranda, J. J., De Wulf, P., Sorger, P. K. \& Harrison, S. C. The yeast DASH complex forms closed rings on microtubules. Nat. Struct. Mol. Biol. 12, 138-143 (2005). 
6. Grishchuk, E. L. et al. The Dam1 ring binds microtubules strongly enough to be a processive as well as energy-efficient coupler for chromosome motion. Proc. Natl Acad. Sci. USA 105, 15423-15428 (2008).

7. Efremov, A., Grishchuk, E. L., McIntosh, J. R. \& Ataullakhanov, F. I. In search of an optimal ring to couple microtubule depolymerization to processive chromosome motions. Proc. Natl Acad. Sci. USA 104, 19017-19022 (2007).

8. Gestaut, D. R. et al. Phosphoregulation and depolymerization-driven movement of the Dam1 complex do not require ring formation. Nat. Cell Biol. 10, 407-414 (2008)

9. Franck, A. D. et al. Tension applied through the Dam1 complex promotes microtubule elongation providing a direct mechanism for length control in mitosis. Nat. Cell Biol. 9, 832-837 (2007).

10. Akiyoshi, B. et al. Tension directly stabilizes reconstituted kinetochoremicrotubule attachments. Nature 468, 576-579 (2010).

11. Sarangapani, K. K., Akiyoshi, B., Duggan, N. M., Biggins, S. \& Asbury, C. L. Phosphoregulation promotes release of kinetochores from dynamic microtubules via multiple mechanisms. Proc. Natl Acad. Sci. USA 110, 7282-7287 (2013).

12. Tien, J. F. et al. Cooperation of the Dam1 and $\mathrm{Ndc} 80$ kinetochore complexes enhances microtubule coupling and is regulated by aurora B. J. Cell Biol. 189, 713-723 (2010)

13. Lampert, F., Hornung, P. \& Westermann, S. The Dam 1 complex confers microtubule plus end-tracking activity to the $\mathrm{Ndc} 80$ kinetochore complex. J. Cell Biol. 189, 641-649 (2010).

14. Miranda, J. J., King, D. S. \& Harrison, S. C. Protein arms in the kinetochoremicrotubule interface of the yeast DASH complex. Mol. Biol. Cell 18, 2503-2510 (2007)

15. Westermann, S., Drubin, D. G. \& Barnes, G. Structures and functions of yeast kinetochore complexes. Annu. Rev. Biochem. 76, 563-591 (2007).

16. Wang, H. W. et al. Architecture of the Dam1 kinetochore ring complex and implications for microtubule-driven assembly and force-coupling mechanisms. Nat. Struct. Mol. Biol. 14, 721-726 (2007).

17. Franck, A. D., Powers, A. F., Gestaut, D. R., Davis, T. N. \& Asbury, C. L. Direct physical study of kinetochore-microtubule interactions by reconstitution and interrogation with an optical force clamp. Methods 51, 242-250 (2010).

18. Powers, A. F. et al. The Ndc80 kinetochore complex forms load-bearing attachments to dynamic microtubule tips via biased diffusion. Cell 136, 865-875 (2009).

19. Asbury, C. L., Gestaut, D. R., Powers, A. F., Franck, A. D. \& Davis, T. N. The Daml kinetochore complex harnesses microtubule dynamics to produce force and movement. Proc. Natl Acad. Sci. USA 103, 9873-9878 (2006).

20. Li, J. M., Li, Y. \& Elledge, S. J. Genetic analysis of the kinetochore DASH complex reveals an antagonistic relationship with the ras/protein kinase A pathway and a novel subunit required for Ask1 association. Mol. Cell Biol. 25, 767-778 (2005).

21. Kastenmayer, J. P. et al. Functional genomics of genes with small open reading frames (sORFs) in S. cerevisiae. Genome Res. 16, 365-373 (2006).

22. Nishimura, K., Fukagawa, T., Takisawa, H., Kakimoto, T. \& Kanemaki, M. An auxin-based degron system for the rapid depletion of proteins in nonplant cells. Nat. Methods 6, 917-922 (2009)

23. Pinsky, B. A., Kung, C., Shokat, K. M. \& Biggins, S. The Ipl1-Aurora protein kinase activates the spindle checkpoint by creating unattached kinetochores. Nat. Cell Biol. 8, 78-83 (2006).

24. Anderson, M., Haase, J., Yeh, E. \& Bloom, K. Function and assembly of DNA looping, clustering, and microtubule attachment complexes within a eukaryotic kinetochore. Mol. Biol. Cell 20, 4131-4139 (2009).

25. De Wulf, P., McAinsh, A. D. \& Sorger, P. K. Hierarchical assembly of the budding yeast kinetochore from multiple subcomplexes. Genes Dev. 17, 2902-2921 (2003).

26. Janke, C. et al. The budding yeast proteins Spc24p and Spc25p interact with $\mathrm{Ndc80} p$ and Nuf2 $\mathrm{p}$ at the kinetochore and are important for kinetochore clustering and checkpoint control. EMBO J. 20, 777-791 (2001).

27. Tanaka, T. U. et al. Evidence that the Ipl1-Sli15 (Aurora kinase-INCENP) complex promotes chromosome bi-orientation by altering kinetochore-spindle pole connections. Cell 108, 317-329 (2002).

28. Koshland, D. E., Mitchison, T. J. \& Kirschner, M. W. Polewards chromosome movement driven by microtubule depolymerization in vitro. Nature 331, 499-504 (1988).

29. Mandelkow, E. M., Mandelkow, E. \& Milligan, R. A. Microtubule dynamics and microtubule caps: a time-resolved cryo-electron microscopy study. J. Cell Biol. 114, 977-991 (1991).

30. Molodtsov, M. I., Grishchuk, E. L., Efremov, A. K., McIntosh, J. R. \& Ataullakhanov, F. I. Force production by depolymerizing microtubules: a theoretical study. Proc. Natl Acad. Sci. USA 102, 4353-4358 (2005).
31. Miller, M. P., Unal, E., Brar, G. A. \& Amon, A. Meiosis I chromosome segregation is established through regulation of microtubule-kinetochore interactions. ELife 1, e00117 (2012).

32. Welburn, J. P. et al. The human kinetochore Skal complex facilitates microtubule depolymerization-coupled motility. Dev. Cell 16, 374-385 (2009).

33. Alushin, G. M. et al. The Ndc80 kinetochore complex forms oligomeric arrays along microtubules. Nature 467, 805-810 (2010).

34. Umbreit, N. T. et al. The Ndc80 kinetochore complex directly modulates microtubule dynamics. Proc. Natl Acad. Sci. USA 109, 16113-16118 (2012).

35. Miller, S. A., Johnson, M. L. \& Stukenberg, P. T. Kinetochore attachments require an interaction between unstructured tails on microtubules and Ndc80(Hec1). Curr. Biol. 18, 1785-1791 (2008).

36. Wei, R. R., Sorger, P. K. \& Harrison, S. C. Molecular organization of the Ndc80 complex, an essential kinetochore component. Proc. Natl Acad. Sci. USA 102, 5363-5367 (2005).

37. Burke, D., Dawson, D. \& Stearns, T. Methods in Yeast Genetics (Cold Spring Harbor Laboratory Press, 2000).

38. Gestaut, D. R., Cooper, J., Asbury, C. L., Davis, T. N. \& Wordeman, L. Reconstitution and functional analysis of kinetochore subcomplexes. Methods Cell Biol. 95, 641-656 (2010).

39. Wargacki, M. M., Tay, J. C., Muller, E. G., Asbury, C. L. \& Davis, T. N. Kip3, the yeast kinesin-8, is required for clustering of kinetochores at metaphase. Cell Cycle 9, 2581-2588 (2010).

40. Straight, A. F., Marshall, W. F., Sedat, J. W. \& Murray, A. W. Mitosis in living budding yeast: anaphase A but no metaphase plate. Science 277, 574-578 (1997).

41. Wach, A., Brachat, A., Alberti-Segui, C., Rebischung, C. \& Philippsen, P. Heterologous HIS3 marker and GFP reporter modules for PCR-targeting in Saccharomyces cerevisiae. Yeast 13, 1065-1075 (1997).

42. Longtine, M. S. et al. Additional modules for versatile and economical PCRbased gene deletion and modification in Saccharomyces cerevisiae. Yeast 14, 953-961 (1998).

43. Muller, E. G. D. et al. The organization of the core proteins of the yeast spindle pole body. Mol. Biol. Cell 16, 3341-3352 (2005).

44. Shimogawa, M. M., Wargacki, M. M., Muller, E. G. \& Davis, T. N. Laterally attached kinetochores recruit the checkpoint protein Bubl, but satisfy the spindle checkpoint. Cell Cycle 9, 3619-3628 (2010).

45. Walker, R. A. et al. Dynamic instability of individual microtubules analyzed by video light microscopy: Rate constants and transition frequencies. J. Cell Biol. 107, 1437-1448 (1988).

\section{Acknowledgements}

The authors thank A. Gupta, E. Mazanka, K. Sarangapani and E. Yusko for their helpful insights. We are grateful to Y. Deng for assistance with Matlab software and L. Chan for providing degron-tagging plasmids used in this study. M.P.M. is an HHMI Fellow of the Damon Runyon Cancer Research Foundation. This work was supported by a National Sciences and Engineering Research Council of Canada scholarship (to J.F.T.), a Packard Fellowship 2006-30521 (to C.L.A.) and NIH grants R01GM064386 (to S.B.), R01GM079373 and S10RR026406 (to C.L.A.) and R01GM40506 (to T.N.D.)

\section{Author contributions}

N.T.U. designed and performed experiments, analyzed the data and wrote the manuscript; M.P.M. generated all yeast strains and assisted with preparation of the manuscript; J.F.T. performed live-cell imaging experiments with N.T.U. and provided feedback on the manuscript; J.C.O. performed in vitro experiments with N.T.U.; L.G. and K.K.L. performed electron microscopy experiments; S.B. provided conceptual advice and assisted in the preparation of the manuscript; C.L.A., and T.N.D. designed experiments and wrote the manuscript with N.T.U.

\section{Additional information}

Supplementary Information accompanies this paper at http://www.nature.com/ naturecommunications

Competing financial interests: The authors declare no competing financial interests.

Reprints and permission information is available online at http://npg.nature.com/ reprintsandpermissions/

How to cite this article: Umbreit, N. T. et al. Kinetochores require oligomerization of Daml complex to maintain microtubule attachments against tension and promote biorientation. Nat. Commun. 5:4951 doi: 10.1038/ncomms5951 (2014). 